\section{Nueva ola de servicios de información}

PARA TODO AQUEL QUE HAYA ESTADO relacionado de alguna forma con el mundo de la información profesional, la presente es una era fascinante. Anteriormente, realizar búsquedas vía electrónica era una verdadera aventura que costaba mucho dinero y requería de una cierta formación en el uso de cada sistema: Questel, Dialog, DataStar, etc., que, aunque existen todavía, ofrecen una forma de acceso algo distinta que en el pasado, principalmente porque con la explosión del web buscar y encontrar información ya no es sólo tarea de expertos.

Pero la calidad de cada buscador es muy variable: desde los que son excelentes hasta los que cuya utilización no aporta ningún beneficio. Lamentablemente no existe un método imparcial para calificarlos. La mejor forma de acreditación es la fama que otorga la satisfacción del usuario, aunque construir algún tipo de índice de calidad objetivo es algo absolutamente necesario. Algo así ya se está haciendo con los grandes buscadores (Yahoo, por ejemplo).

Desde hace algunos años se realiza un estudio sobre la cobertura de estas herramientas. El publicado en el número de julio de 1999 de Nature indica que el buscador con más cobertura, NorthernLight, cubre sólo el 16\% de los 800 millones de páginas web que se estima que existen. Muy cerca le sigue Altavista $(15,5 \%)$ mientras que $\mathrm{Ya}$ - infoUSA $\mathrm{Z}$ com hoo alcanza el 7,4\% —aunque hay que recordar que en éste la indización es, en buena parte, humana y se supone de mayor calidad-.

http://www.northernlight.com/docs/in telligent_stats.htmlhttp://www.notess. $\mathrm{com} /$ search/stats/sizeest.shtml

Hay otro tipo de directorios que buscan crearse un espacio. Es el caso de GuiaCom en España o InfoPime en
Perú, ambos dedicados al mundo de la empresa y los negocios.

http://www.guiacom.es/menufijo.html

http://www.superviaperu.com/infopimel

Pero lo más interesante consiste en la aparición de sistemas cuya oferta amplifica el valor informacional de manera importante. Un buen ejemplo son aquellos que permiten consultar publicaciones diversas en texto completo. En este sentido, es especialmente interesante el servicio brasileño Scielo, que ofrece acceso gratuito a decenas de revistas científicas y técnicas brasileñas, una iniciativa que debería ser imitada. Otro caso, aunque sea de pago, es EngNet cuya especialidad es la ingeniería.

http://www.scielo.br/

http://www.engnetbase.com/

En países informacionalmente más avanzados aparecen cada día propuestas más y más sofisticaUIACOM das que persiguen responder a ortal Empresarial necesidades cada vez más concretas. Por ejemplo, en UsaData, las empresas pueden encontrar una gran cantidad de tablas con información sobre estudios de mercado, con acceso por medio del pago por documento. El sistema permite construir dicha tabla a medida, combinando la información precisa que uno necesita a partir de los datos básicos almacenados en la base de datos principal. Otro sistema interesante es InfoUSA, donde es posible conseguir mailings, listados procedentes de distintos sistemas de páginas amarillas, etc.

\section{http://www.usadata.com/}

http://www.infousa.com/

Pero quizás lo más interesante es el DotCom directory. Una iniciativa de NetWorkSolutions, la empresa que gestionaba hasta ahora en exclusiva los dominios .com y que, posiblemente, sea la mayor base de datos de empresas del mundo.

Alfons Cornella

\section{SUMARIO}

\section{Articulos}

Posibles escenarios para las industrias digitales Por Francesc Mañà .......................................................

Pagos, micropagos e internet

Por Cristina García Testalz

Observatorio

Análisis de la producción de El profesional

de la información ..........................................................20

\section{Mercado}

La Biblioteca Rusa se automatiza ……………………...23 Conservación digital del patrimonio cultural ....................24 Consultoría bibliotecaria en Argentina ..............................25

Claves del éxito de los diarios económicos españoles en internet. Por Ramón Salaverría ………………………...26

\section{Recursos informativos en las redes}

Página web de recursos médicos Por Roberto Juan Falagán...

\section{sistemas de informacion}

Nueva ola de servicios de información ………................... Desarrollos en buscadores ..............................................32 El rol del infonomista . .34 ¿Afectará la crisis económica al comercio electrónico? ·36 Dos estrategias básicas en la gestión del conocimiento $\cdot 38$ Metamediarios y metamercados

\section{Resenas}

Segundo forum internacional sobre la gestión de los documentos electrónicos. Por Jordi Serra Serra ......................40 El «mejor artículo» de 1999..............................................44 Recursos electrónicos y catálogo ………………………44

\section{Agenda} 50

Informacion para los autores

Los trabajos de la sección Artículos son aprobados según el sistema tradicional "peer review": al menos dos expertos en el tema, del Consejo Asesor de la revista y/o externos, deben dar el visto bueno antes de su publicación. 